\title{
Application of low-order Discontinuous Galerkin methods to the analysis of viscoelastic flows
}

\section{Frank P.T. Baaijens}

Philips Research Laboratories, P.O. Box 80000, 5600 JA Eindhoven (The Netherlands) and Eindhoven University of Technology, Center for Polymers and Composites, P.O. Box 513, $5600 \mathrm{MB}$ Eindhoven (The Netherlands)

(Received September 13, 1993; in revised form November 30, 1993)

\begin{abstract}
The performance of two low-order discretization schemes in combination with the Discontinuous Galerkin method for the analysis of viscoelastic flows is investigated. An (extended) linear interpolation of the velocitypressure variables is used in combination with a piecewise discontinuous constant and linear approximation of the extra stresses. Galerkin-leastsquares methodology is applied to stabilize the velocity-pressure discretization. As test problems, the falling sphere in a tube and the stick-slip configuration are studied. The constant stress triangular element converges to high Deborah numbers for a wide variety of material parameters of the Phan-Thien-Tanner model. In particular, for the upper convected Maxwell model, the falling sphere problem converges at least up to Deborah number of 4, while the stick-slip problem converges up to a Deborah number of 25.5.
\end{abstract}

Keywords: Discontinuous Galerkin methods; falling sphere problem; Phan-Thien-Tanner model; stabilization; stick-slip problem; viscoelastic flow

\section{Introduction}

Most mixed (stress-velocity-pressure) formulations for viscoelastic flows employ continuous interpolations of the independent variables [1-10]. The compatibility requirements on the stress-velocity interpolation are not easily met, in particular at vanishing solvent viscosity (Baranger and Sandri [11]), and may lead to complicated elements [3]. For discontinuous interpolations of the extra stresses compatibility is easily satisfied (see Ying [12] and Fortin and Pierre [13]). This has led Fortin and coworkers [14,15] to 
apply the Discontinuous Galerkin method (DG) to handle the advective parts of the constitutive equation.

An alternative approach to handle discontinuous interpolations of the extra stress variables was shown by Baaijens $[16,17]$ who used an operator splitting method. This algorithm, however, only applies to unsteady problems.

The most successful implementation of the DG method is obtained by combining DG with the so-called Elastic Viscous Split method (EVS), as originally proposed by Medelson et al. [18], and is found in Fortin et al. [15]. In analysing the stick-slip problem with the Phan-Thien-Tanner constitutive model, convergence, however, is not obtained for all values of the Deborah number, in particular when approaching the upper convected Maxwell model limit. This is most likely due to the second-order interpolation of the extra stress tensor that has been employed. For this reason, Basombrio et al. [19] investigated a linear discretization of the stress field on triangles. The underlying velocity element contains four linear triangular elements, while a linear approximation of the pressure field is used (see also Pironneau [20]). High values of elasticity have been reached in the four-toone contraction problem using the Oldroyd-B model. It was shown in Baaijens [21] that, in an unsteady formulation, stress oscillations appearing in the linearly interpolated scheme may be effectively controlled by using monotonicity enforcement.

The key objective of this work is to study the performance of a piecewise linear and constant approximation of the extra stress tensor in conjunction with an (extended) linear interpolation (on triangles) for both the velocity and pressure field for steady viscoelastic flows.

Stability of the velocity-pressure discretization for equal-order linear triangular elements is achieved by employing the stabilization technique proposed by Franca and Stenberg [22], Behr et al. [23] and Tezduyar et al. [24] based on principles developed by Hughes et al. [25]. The low-order interpolation produces a robust algorithm, giving convergence at high values of the Deborah number $(D e)$ for a wide variety of the material parameters of the Phan-Thien-Tanner (PTT) model for both smooth and non-smooth problems, even in the limit of the upper convected Maxwell (UCM) model.

A number of numerical tests are carried out. Firstly, the steady flow about a falling sphere in a tube is examined. This is a so-called smooth test problem and has been investigated in detail by, among others, Crochet and Legat [26], Rasmussen and Hassager [27], Chilcott and Rallison [28], Harlen [29], and more recently by Lunsmann et al. [30]. This problem is characterized by a pronounced stress boundary layer structure at the edge of the sphere, combined with a strong elongational flow along the symmetry line after the sphere. These fine stress features are difficult to capture numerically. Using the UCM model, the constant stress element gives a 
convergent Newton iteration process up to a Deborah number of at least 4 . This is remarkable as others, see Refs. 26 and 30, report a loss of convergence of the iterative scheme beyond $D e \approx 1.6$. Convergence to higher values of $D e$ is obtained when use is made of non-linear models $[28,30]$.

Secondly, the stick-slip problem is considered, which is known to be very difficult to solve. This is a representative of the class of non-smooth problems as it contains a singularity induced by the transition from stick to slip boundary condition. Many algorithms fail to converge at moderate values of elasticity and significant oscillations in the stress fields are usually observed eventually leading to a loss of convergence of the iterative scheme $[3,15,21,31,22]$.

The outline of this paper is as follows. Firstly, the problem definition is given, after which the stabilized Discontinuous Galerkin method is discussed. The performance of the method is then illustrated by the analysis of the falling sphere and the stick-slip problems. Finally, conclusions are drawn.

\section{Problem definition}

Consider the steady flow of a PTT fluid in a two-dimensional domain $\Omega$ with boundary $\Gamma$, defined by:

Problem 1 (PVE) Given $\vec{u}^{0}: \Gamma_{u} \mapsto \mathbb{R}^{2}, \tau^{0}: \Gamma_{\text {in }} \mapsto \mathbb{R}^{2 \times 2}$ and $\vec{t}^{0}: \Gamma_{\tau} \mapsto \mathbb{R}^{2}$, find the plane or axisymmetric stress field $\tau(\vec{x}): \Omega \mapsto \mathbb{R}^{(2 \times 2)}$, the velocity field $\vec{u}(\vec{x}): \Omega \mapsto \mathbb{R}^{2}$ and the pressure field $p(\vec{x}): \Omega \mapsto \mathbb{R}$ for all $\vec{x} \in \Omega$, such that

$\vec{\nabla} \cdot\left(-p I+2 \eta_{0} D+\tau\right)=\overrightarrow{0}$,

$\vec{\nabla} \cdot \vec{u}=0$,

with for the PTT model

$\stackrel{\tau}{\tau}+\left[\frac{1}{\lambda}+\frac{\epsilon}{\eta} \operatorname{tr}(\tau)\right] \tau=\frac{2 \eta}{\lambda} D$,

where $\stackrel{\nabla}{\tau}=\vec{u} \cdot \vec{\nabla} \tau-L \cdot \tau-\tau \cdot L^{\mathrm{T}}$, with $\boldsymbol{L}=(\vec{\nabla} \vec{u})^{\mathrm{T}}$ and $2 \boldsymbol{D}=\boldsymbol{L}+\boldsymbol{L}^{\mathrm{T}}$, while the following boundary conditions are specified on $\Gamma$

$\vec{u}(\vec{x})=\vec{u}^{0}(\vec{x})$ on $\Gamma_{u}$,

$\left[-p I+2 \eta_{0} D+\tau(\vec{x})\right] \cdot \vec{n}=\vec{t}^{0}(\vec{x})$ on $\Gamma_{\tau}$

and along the inflow boundary

$\tau=\tau^{0} \quad$ on $\Gamma_{\text {in }}$, 
with $\Gamma=\Gamma_{u} \cup \Gamma_{\tau}, \vec{n}$ the unit outward normal at $\Gamma_{\imath}$, and $\Gamma_{\text {in }}$ the inflow boundary: the part of $\Gamma$ where $\overrightarrow{\mathbf{u}} \cdot \vec{n}<0$.

The material parameters in the above PTT model are: the solvent viscosity, denoted by $\eta_{0}$, the relaxation time, denoted by $\lambda$, the viscosity, denoted by $\eta$, while $\epsilon$ denotes a dimensionless parameter characteristic for this particular PTT model. For non-zero $\epsilon$ this model has a shear thinning viscosity and a bounded elongational viscosity. This is in contrast with the limit of $\epsilon=0$, where the model reduces to the UCM model. This model has a constant shear viscosity and an unbounded elongational viscosity when $\lambda \dot{\epsilon}=1 / 2$, where $\dot{\epsilon}$ is the uniaxial elongational rate. The lack of convergence of the numerical schemes for the analysis of flows with the UCM model is sometimes attributed to this unbounded elongational viscosity $[10,33]$.

\section{Stabilized Discontinuous Galerkin method}

Recently, Franca and Stenberg [22] introduced a stabilized stress velocity-pressure formulation for the Stokes problem that allows for various combinations of interpolation schemes of these variables; see also Refs. 23-25 and 34. In particular, it allows for an equal-order interpolation of the velocity and pressure fields. Using a linear interpolation on triangles for these fields, it is possible to use a piecewise constant discontinuous interpolation of the extra stress tensor. This gives a very stable algorithm, as is shown in the sequel.

Define $\mathscr{L}_{\tau}$ as

$\mathscr{L} \tau=\vec{u} \cdot \vec{\nabla} \tau-L \cdot \tau-\tau \cdot \mathbf{L}^{\mathrm{T}}+\left[\frac{1}{\lambda}+\frac{\epsilon}{\eta} \operatorname{tr}(\tau)\right] \tau$

and set

$\mathscr{R}=\vec{\nabla} \cdot\left(-p I+2 \eta_{0} D+\tau\right)$.

Then, assuming a discontinuous interpolation of the extra stress tensor from the onset, application of the Discontinuous Galerkin (DG) method and generalization of the stability concepts of Franca and Stenberg [22], gives

Problem 2 (DG) Find $(\tau, \vec{u}, p) \in \mathscr{S} \times \mathscr{U} \times \mathscr{Q}$, such that for all $(s, \vec{v}, q) \in \mathscr{S} \times \mathscr{V} \times \mathscr{Q}$

$$
\begin{gathered}
\left(s, \mathscr{L} \tau-2 \frac{\eta}{\lambda} \boldsymbol{D}_{u}\right)+\sum_{e=1}^{N_{\mathrm{el}}}\left[(\delta \vec{\nabla} \cdot s, \overrightarrow{\mathscr{R}})_{e}-\int_{\Gamma_{\mathrm{in}}} s: \vec{u} \cdot \vec{n}\left(\tau-\tau^{\mathrm{ext}}\right) \mathrm{d} \Gamma\right]=0, \\
-\left(\boldsymbol{D}_{v}, 2 \eta_{0} \boldsymbol{D}_{u}+\tau\right)+(\vec{\nabla} \cdot \vec{v}, p)+\sum_{e=1}^{N_{\mathrm{el}}}\left(\delta 2 \eta_{0} \vec{\nabla} \cdot \boldsymbol{D}_{v}, \overrightarrow{\mathscr{R}}\right)_{e}=0
\end{gathered}
$$


$(q, \vec{\nabla} \cdot \vec{u})-\sum_{e=1}^{N_{\text {el }}}(\delta \vec{\nabla} q, \overrightarrow{\mathscr{R}})_{e}=0$.

In this, $(.,$.$) and (., .)_{e}$ denote the appropriate inner product on $\Omega$ and $\Omega^{e}$, respectively; $\Gamma_{\text {in }}^{e}$ is the inflow boundary element $\Omega^{e}, \vec{n}$ the unit outward normal to $\Gamma^{e}, \tau^{\text {ext }}$ the stress tensor of the neighbouring, upwind, element, $\delta$ is a stability constant defined below, $\boldsymbol{D}_{u}=\boldsymbol{D}(\vec{u})$, and $\boldsymbol{D}_{v}=\boldsymbol{D}(\vec{v})$.

The terms containing the parameter $\delta$ are obtained by adding a leastsquares term related to the equilibrium equation, expressed by $\overrightarrow{\mathscr{R}}=\overrightarrow{0}$, to the original weak formulation; see Franca and Stenberg [22] for details. The purpose of these terms is to circumvent the so-called inf-sup or BabuskaBrezzi criterion, thereby admitting a wider range of velocity-pressure combinations than normally allowed.

Spatial discretization is accomplished as follows. The domain $\Omega$ is divided into $N_{\text {el }}$ elements such that $\Omega=\cup_{e=1}^{N_{\text {el }}} \Omega^{e}$. Denote with $P_{k}\left(\Omega^{e}\right)$ and $Q_{k}\left(\Omega^{e}\right)$ with $k$ th order interpolation polynomial on a triangular and quadrilateral element $e$, respectively. Define $R_{k}$ by

$R_{k}\left(\Omega^{e}\right)=\left\{\begin{array}{l}P_{k}\left(\Omega^{e}\right) \text { on triangles, } \\ Q_{k}\left(\Omega^{e}\right) \text { on quadrilaterals. }\end{array}\right.$

In a more generalized sense, $P_{k}$ represents linear, quadratic, etc. discretization, while a $Q_{k}$ discretization contains higher-order terms than strictly implied by the order $k$.

The finite element spaces $\mathscr{U}, \mathscr{V}, \mathscr{Q}$ and $\mathscr{S}$ are defined by

$\mathscr{U}=\left\{\vec{u} \in\left[H^{1}(\Omega)\right]^{2}|\vec{u}|_{\Omega^{e}} \in\left[R_{k}\left(\Omega^{e}\right)\right]^{2}, \vec{u}=\vec{u}^{0}\right.$ on $\left.\Gamma_{u}\right\}$,

$\mathscr{V}=\left\{\vec{v} \in\left[H^{1}(\Omega)\right]^{2}|\vec{v}|_{\Omega^{e}} \in\left[R_{k}\left(\Omega^{e}\right)\right]^{2}, \vec{v}=\overrightarrow{0}\right.$ on $\left.\Gamma_{u}\right\}$,

$\mathscr{Q}=\left\{q \in L_{2}(\Omega)|q|_{\Omega^{e}} \in R_{l}\left(\Omega^{e}\right)\right\}$,

$\mathscr{S}=\left\{s \in\left[L_{2}(\Omega)\right]^{3 \times 3}|s|_{\Omega^{e}} \in\left[R_{k}\left(\Omega^{e}\right)\right]^{3 \times 3}\right\}$.

In this paper the $(s, \vec{u}, p) \rightarrow Q_{2}^{d} Q_{2} P_{1}^{d},(s, \vec{u}, p) \rightarrow P_{1}^{d} P_{1}^{+} P_{1}$ and the $(s, \vec{u}, p) \rightarrow P_{0}^{d} P_{1} P_{1}$ discretizations are employed, see Fig. 1 . The superscript $d$ denotes a discontinuous interpolation, and $P_{1}^{+}$denotes an extended linear interpolation achieved by adding a so-called bubble function to the regular $P_{1}$ discretization; see Pironneau [20].

It is tempting to include the $Q_{0}^{d} Q_{1} Q_{1}$ element in this study. With a non-zero $\delta$ parameter it does give a stable pressure solution, but the constant stress approximation is incompatible with the bilinear velocity approximation. The necessary requirement $\nabla \mathscr{V} \subset \mathscr{S}$ is not fulfilled for this element. This also holds for the $P_{0}^{d} P_{1}^{+} P_{1}$ element.

The first two discretization schemes $\left(Q_{2}^{d} Q_{2} P_{1}^{d}\right.$ and $\left.P_{1}^{d} P_{1}^{+} P_{1}\right)$ do not need additional stability induced by the $\delta$ parameter, they satisfy the inf - sup 

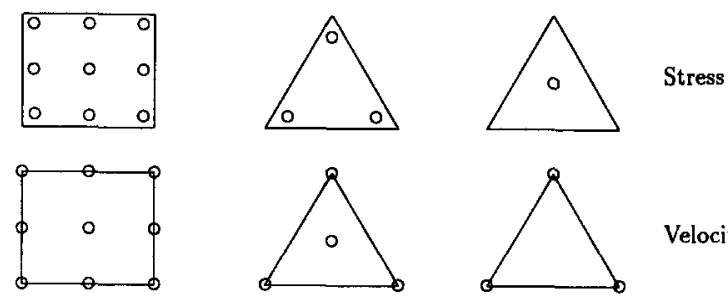

Velocity
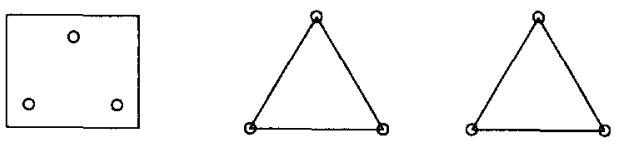

pressure

$Q_{2}^{d} Q_{2} P_{1}^{d}$

$P_{1}^{d} P_{1}^{+} P_{1}$

$P_{0}^{d} P_{1} P_{1}$

Fig. 1. Discretization schemes.

condition on the velocity-pressure discretization if $\delta=0$, hence, $\delta$ is taken equal to zero for these interpolation schemes. This is not the case for the $P_{0}^{d} P_{1} P_{1}$ element. In that case it was shown by Behr et al. [23] that for viscous flows a stable method is obtained with

$\delta=\frac{h_{e}^{2}}{32 \eta}, \quad h_{e}=\sqrt{\operatorname{area}\left(\Omega^{e}\right)}$.

For viscoelastic flows with the PTT model, the above is generalized to

$\delta=\frac{h_{e}^{2}}{32 \bar{\eta}}, \quad \bar{\eta}=\frac{\eta^{2}}{\eta+\epsilon \theta \operatorname{tr}(\tau)}$.

For this constant stress, linear velocity element, $\vec{\nabla} \cdot D_{v}=\overrightarrow{0}$ and $\vec{\nabla} \cdot s=\overrightarrow{0}$, and it follows that the inner products containing the $\delta$ parameter are identically zero in eqns. (9) and (10), while eqn. (11) reduces to

$(q, \vec{\nabla} \cdot \vec{u})+(\vec{\nabla} q, \delta \vec{\nabla} p)=0$.

This is the only modification to the weak formulation compared to the formulation without the added least-squares terms.

After discretization, a set of non-linear equations emerges. In the first implementation of the DG method by Fortin and Fortin [14] a time-stepping scheme was applied in conjunction with a Picard iteration process. Later, after combining DG with a change of variables known as the Elastic Viscous Split method, recourse is made to the GMRES algorithm, [35,15], giving a more Newton-like convergence behaviour. In that case, the Jacobian matrix is approximated by a block diagonal matrix, in the sense that cross derivatives of eqns (9) and (10) are not taken into account. The key advantage of this is that the extra stress variables may be eliminated on the 
element level. Subsequently, the velocity-stress coupling is induced by the GMRES iteration.

In this work, however, a Newton iteration procedure is adopted. The only non-differentiable term is the integral over the element inflow boundary $\Gamma_{\text {in }}^{e}$ and consequently this boundary is evaluated at the previous iteration level. This procedure did not significantly upset the convergence behaviour in the test problems. The linear set of equations is solved with a direct sparse matrix solver as implemented in MATLAB [36].

\section{Numerical experiments}

Two flow situations are investigated: the axisymmetric flow around a sphere in a tube having pronounced boundary layers at the sphere surface and the plane stick-slip problem containing a singularity.

Unless specified otherwise, the solvent viscosity $\eta_{0}$ is set to zero in subsequent computations, and the Deborah number is taken as $D e=\lambda \dot{\gamma}_{\mathrm{w}}$, with $\dot{\gamma}_{w}$ the shear rate at the wall.

In a previous study of the DG method, Fortin and Fortin [14] considered the four-to-one contraction problem and used the $Q_{2}^{d} Q_{2} P_{1}^{d}$ element. Oscillation-free solutions beyond $D e=1.6$ for the Oldroyd-B model were only obtained by introduction of additional streamline diffusion. Basombrio et al. [19] also investigated the DG method in combination with the Oldroyd-B model, using a linear stress interpolation on four linear velocity sub-triangles, for the four-to-one contraction problem and obtained convergent results up to $D e=18$. The stick-slip problem was chosen as a test problem by Fortin et al. [15]. Using the PTT model, good results are obtained for $\epsilon=0.25$, but, decreasing $\epsilon$, and thereby increasing the elongational viscosity, leads to a loss of convergence.

The flow about a sphere at the axis of the tube is a standard test problem, and is examined in detail, among others, by Crochet and Legat [26], Rasmussen and Hassager [27] and Lunsmann et al. [30]. In general, for the UCM model convergence is reached up to $D e \approx 1.6$.

The stick-slip problem with a UCM model was also investigated by Armstrong et al. [31], who found convergent results up to $D e=0.6$. Using the consistent SUPG method with an Oldroyd-B model, Marchal and Crochet [3] obtained convergence up to $D e=10$, but the stress field showed unacceptable oscillations. Using the inconsistent SU method, however, convergence is reached to at least $D e=27$ and smooth stress fields are obtained. But, there is some doubt regarding the accuracy of the SU method; see Crochet and Legat [26] and Tanner and Jin [37]. The method is first-order accurate but large consistency errors may exist, requiring highly refined meshes. 


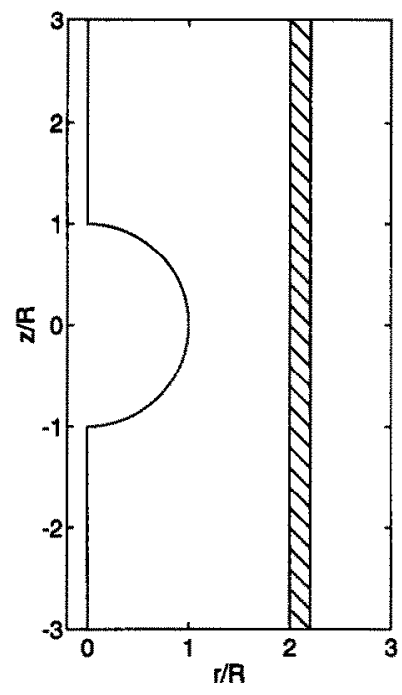

Fig. 2. Flow domain; sphere has radius $R$ and tube has radius $R_{t}$, the upstream and downstream tube lengths are $L_{1}$ and $L_{2}$, respectively. In all computations $R_{\mathrm{t}} / R=2, L_{1} / R=5$ and $L_{2} / R=15$.

\subsection{Flow around a sphere in a tube}

The steady flow around a sphere placed at the centreline of a tube is investigated. The tube wall moves parallel to the centerline with a velocity $V$ in the positive $z$-direction. The flow domain is sketched in Fig. 2. The ratio of the tube radius $R_{\mathrm{t}}$ and the sphere radius $R, \chi=R_{\mathrm{t}} / R=2$.

The Deborah number is defined as

$$
D e=\frac{\lambda V}{R} \text {. }
$$

The drag $F$ on a sphere falling in an unbounded Newtonian medium is given by

$F=6 \pi \eta R V$.

An approximation for a sphere falling through a tube filled with a Newtonian fluid is computed by Happel and Brenner [38], and is given by a wall correction factor $K(\chi)$ (Lunsmann et al. [30])

$K(\chi)=\frac{F(\chi)}{6 \pi \eta R V}$, 
with

$$
\begin{aligned}
\eta R V K(\chi) \approx & \left(1-2.1044 \chi^{-1}+2.08877 \chi^{-3}-0.94813 \chi^{-5}\right. \\
& \left.-1.372 \chi^{-6}+3.87 \chi^{-8}-4.19 \chi^{-10}+\cdots\right)^{-1}
\end{aligned}
$$

which, formally, only holds for $\chi \ll 1$. For $\chi=2, K=5.9229$.

First, this semi-analytical solution is compared with numerically predicted drag correction factors at Newtonian flow conditions. This gives an impression of the relative accuracy that may be expected. Subsequently, viscoelastic flow of the UCM model is investigated.

\subsubsection{Newtonian flow}

The Newtonian problem is solved utilizing the $P_{0}^{d} P_{1} P_{1}$, the $P_{1}^{d} P_{1}^{+} P_{1}$ and the $Q_{2}^{d} Q_{2} P_{1}^{d}$ elements. For the constant and linear stress elements, three meshes have been used, meshes $1-3$, with mesh characteristics listed in Table 1. For the quadrilateral element meshes 5 and 6 have been used. The mesh size parameter $h_{\text {min }}$ denotes the length of the element side in the radial direction located at $r / R=1, z / R=0$. Details of mesh 1 and mesh 4 are shown in Fig. 3.

Meshes 2 and 3 are uniform refinements of mesh 1, while meshes 5 and 6 have a similar structure as mesh 1. Mesh 4 , on the other hand, has a different structure, see Fig. 3.

The drag force $F$ is computed by summation of the reaction forces of the nodes on the sphere, which result from the residual of eqn. (10) after imposing the prescribed displacements. The computed drag correction factor $K$ as a function of $h_{\min }$ is shown in Fig. 4. The dashed line denotes the analytical value. Little difference is found between the constant and linear triangular stress elements $\left(P_{0}^{d} P_{1} P_{1}\right.$ and $P_{1}^{d} P_{1}^{+} P_{1}$, respectively). The quadratic element is clearly more accurate, as may be expected. The

\section{TABLE 1}

Characteristic mesh parameters. The numbers between brackets refer to the linear stress element

\begin{tabular}{llcll}
\hline Mesh & $h_{\min } / R$ & No. of elements & No. of nodes & $\begin{array}{l}\text { No. of degrees } \\
\text { of freedom }\end{array}$ \\
\hline 1 & 0.0203 & 518 & $304(822)$ & $2984(8682)$ \\
2 & 0.0147 & 980 & $550(1530)$ & $5570(16350)$ \\
3 & 0.01 & 2280 & $1232(3512)$ & $12816(37896)$ \\
4 & 0.01 & 1950 & $1056(3006)$ & $10968(32418)$ \\
5 & 0.0147 & 490 & 2029 & 23168 \\
6 & 0.001 & 1035 & 4309 & 48983 \\
\hline
\end{tabular}



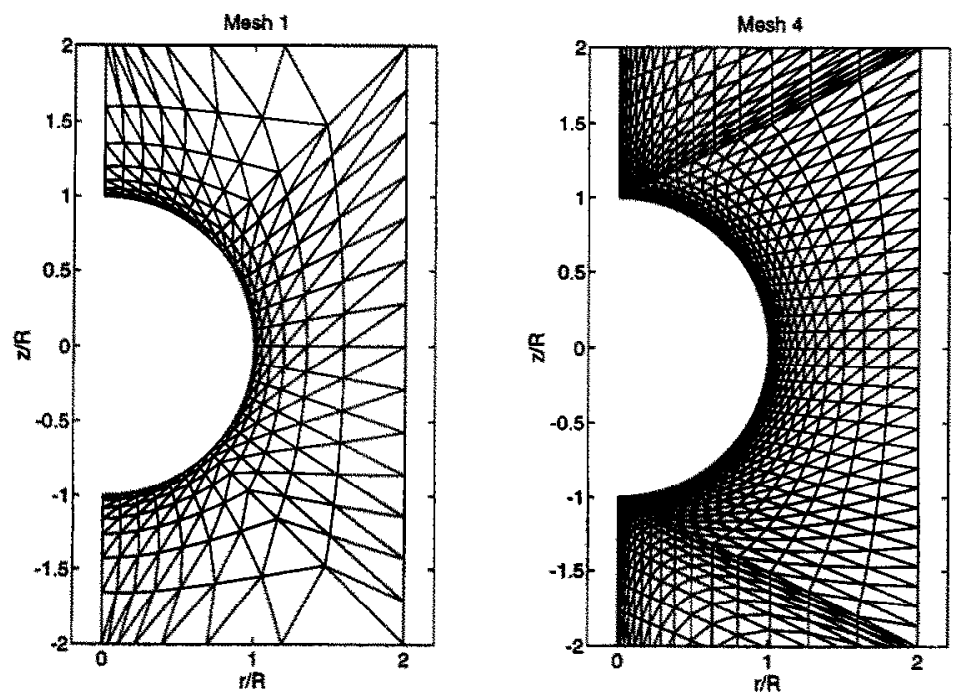

Fig. 3. Detail of mesh 1 and mesh 4 near the sphere.

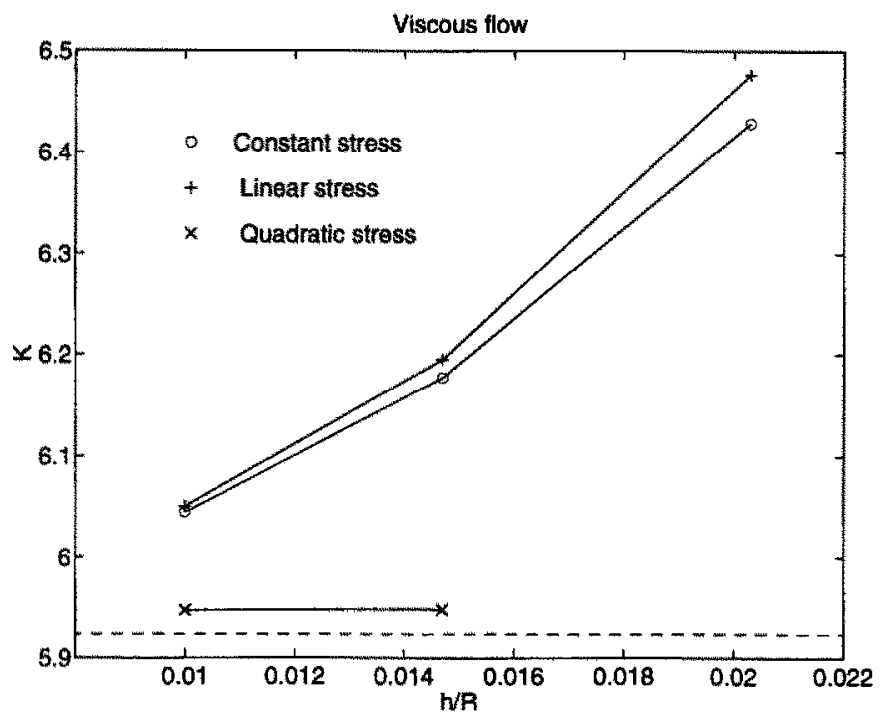

Fig. 4. Drag correction factor $K$ as a function of the mesh size. The o symbols correspond to element $P_{0}^{d} P_{1} P_{1}$, the + symbols correspond to element $P_{1}^{d} P_{1}^{+} P_{1}$ and the $\times$ symbols correspond to element $Q_{2}^{d} Q_{2} P_{1}^{d}$. The dashed line represents the analytical result.

numerical prediction does not exactly correspond to the theoretical result of eqn. (23) as this last value is an approximation that only holds for $\chi \ll 1$, which is not the case here. Compared to the results presented by Lunsmann et al. [30], it is remarkable to see that the theoretical value is approached 
from above, rather than from below. The cause of this phenomenon is unclear.

\subsubsection{Viscoelastic flow of a UCM fluid}

For the viscoelastic problem, meshes 1, 2 and 4 are used. More refined meshes could not be experimented with due to memory limitations of our software/hardware configuration. It may be clear from the Newtonian computations that this will only give limited accuracy.

Clearly, to obtain the UCM model, the $\delta$ parameter in the PTT model is set to zero. In Fig. 5 the computed drag correction factor for the constant $\left(P_{0}^{d} P_{1} P_{1}\right)$ and linear stress $\left(P_{1}^{d} P_{1}^{+} P_{1}\right)$ elements are compared with the results of Lunsmann et al. [30].

Starting at the Newtonian solution, the Deborah number is incremented by 0.1 or 0.2 until 3.0 (or higher) where possible. In all convergent increments a quadratic rate of convergence is observed. The linear stress element exhibits a convergent Newton iteration process only up to $D e=1.6$ on mesh 1 (+symbols in Fig. 5). More refined meshes could not be tested due to memory limitations. The constant stress element, on the other hand, shows a convergent Newton iteration process at least up to $D e=4.0$ on all meshes. This is quite remarkable as Crochet and Legat [26], Rasmussen and Hassager [27] and Lunsmann et al. [30] report a loss of convergence of the Newton iteration scheme beyond $D e \approx 1.6$ when using the UCM model. Furthermore they observe convergence to higher values of the Deborah number with increasing mesh refinement, whereas the current method gives

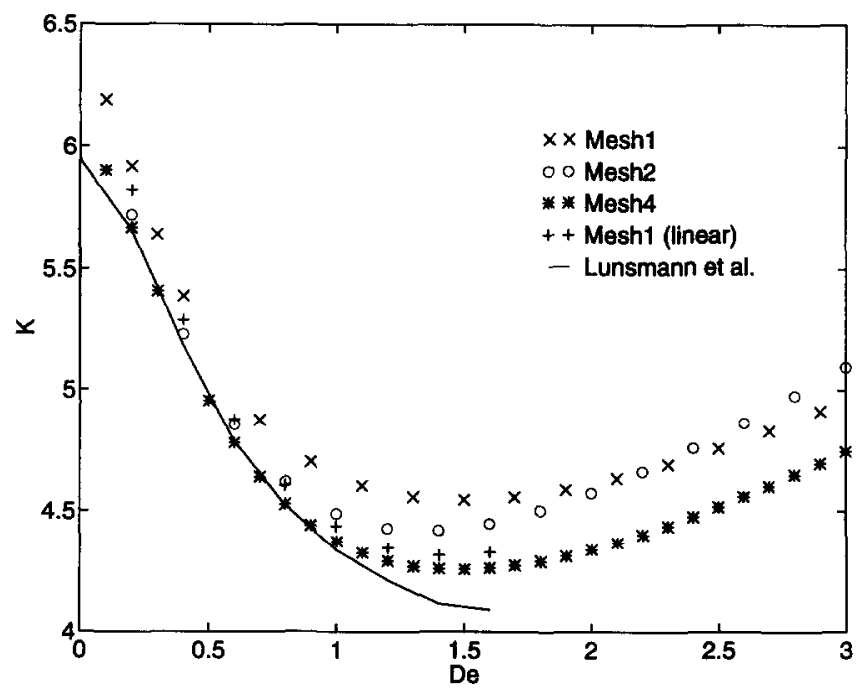

Fig. 5. Drag correction factor $K$ as a function of the Deborah number. 


\section{TABLE 2}

Drag correction factor as a function of the Deborah number for mesh 4 compared to the results of Lunsmann et al. [30] (EVSS) and of Crochet and Legat [26] (SU4 × 4)

\begin{tabular}{llll}
\hline De & $\begin{array}{l}\text { EVSS } \\
(28558)\end{array}$ & $\begin{array}{l}\text { Mesh 4 } \\
(10968)\end{array}$ & $\begin{array}{l}\text { SU4 } \times 4 \\
(75512)\end{array}$ \\
\hline 0.2 & 5.658 & 5.667 & 5.654 \\
0.4 & 5.180 & 5.165 & 5.192 \\
0.6 & 4.788 & 4.780 & 4.825 \\
0.8 & 4.523 & 4.527 & 4.569 \\
1.0 & 4.336 & 4.374 & 4.406 \\
1.2 & 4.211 & 4.291 & 4.312 \\
1.4 & 4.133 & 4.258 & 4.266 \\
1.6 & 4.088 & 4.261 & 4.258 \\
\hline
\end{tabular}

convergence of the Newton iteration process at high values of the Deborah number for all meshes.

Table 2 gives a quantitative comparison of the predicted drag correction factor on mesh 4 with the DG method using the constant stress element, with the results of Lunsmann et al. [30], based on their Elastic Viscous Split Stress (EVSS) formulation, and the predictions of Crochet and Legat [26], based on the SU $4 \times 4$-formulation. The numbers in brackets denotes the number of degrees of freedom involved in the analysis. It must be remarked that Lunsmann et al. [30] also produced quite accurate results using the Explicitly Elliptic Momentum Equation (EEME) method with only 8329 degrees of freedom, while the use of SUPG $4 \times 4$ by Crochet and Legat [26] gave correspondence with the EVSS results up to the first and/or second decimal.

The linear stress element is clearly more accurate than the constant stress element; this is in contradiction to the Newtonian results of Fig. 4, where the constant and linear stress elements produced almost identical results.

After an initial decrease, a subsequent increase in the drag correction factor is seen. The computed drag correction factor $K$ seems to reach a minimum at $D e \approx 1.6$. However, it is clear that convergence with mesh refinement is not established. Therefore, it is not clear at present if this apparent minimum is an artefact of the limited accuracy of the current computation or not. The streamline upwind results of Crochet and Legat [26] (SU4 $\times 4$ ) also show a minimum in the drag correction curves. This minimum, however, is not present in the more accurate streamline-upwind Petrov-Galerkin results. This supports the argument that this minimum is due to computational inaccuracies. Unfortunately, mesh 4 is the most refined mesh currently permitted by our computational system. 

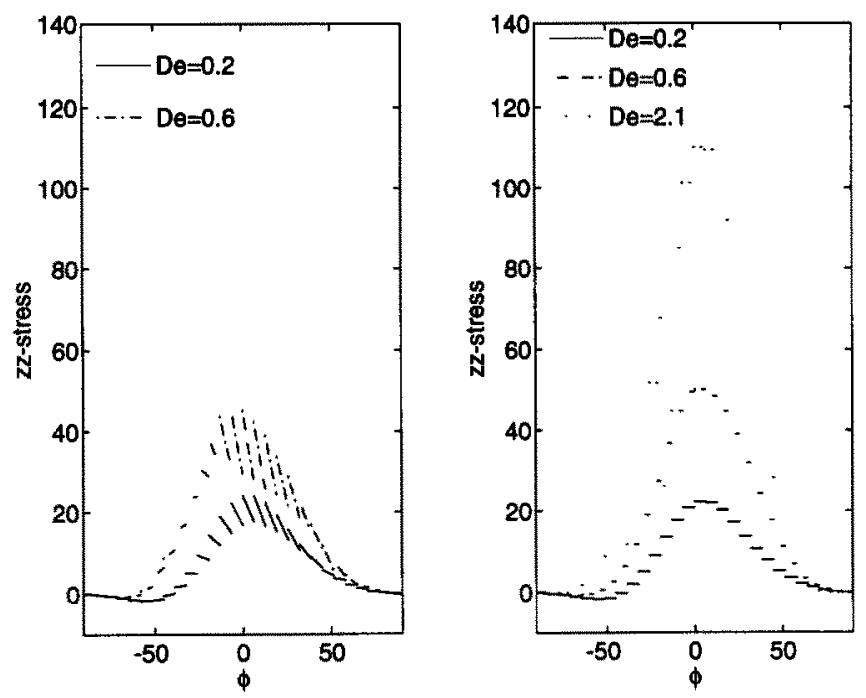

Fig. 6. $\tau_{z z}$ along the sphere boundary as function of the polar angle $\phi$, starting at the upstream axis. Left figure for the linear stress element at $D e=0.2$ and $D e=0.6$; right figure for the constant stress element at $D e=0.2,0.6$ and 2.1.

But, on the whole, a good qualitative correspondence is observed between the current results and the values of Lunsmann et al. [30]. The quantitative differences are attributed to discretization errors, as in the Newtonian case. For the constant stress element, the number of elements should exceed 10000 to have resolution approximately equal to Lunsmann et al. [30] at $D e>1.0$. This may also be seen from the results of the Newtonian analysis, see Fig. 4. This is, unfortunately, beyond practical capabilities of our software/hardware configuration.

The $\tau_{z z}$ stress component along the sphere is plotted in Fig. 6, as a function of the polar angle starting at the upstream stagnation point. Both the results for the linear and constant stress elements for mesh 1 are displayed. Already at $D e=0.2$ the linear stress element shows vivid oscillations which eventually lead to the loss of convergence of the Newton iteration process. These oscillations, however, are confined to almost the first row of elements at the edge of the sphere. The constant stress element, on the other hand, shows a smooth monotonic increase of $\tau_{z z}$. Other stress components behave similarly. Surprisingly, the oscillations in the stress field of the linear stress element do not seem to upset the accuracy of the predicted drag correction factor (Fig. 5). Most likely this implies that the average value of the extra stress variables is reasonably accurate.

For qualitative comparison, contour lines of the $\tau_{z z}$ component for the constant stress element at various Deborah numbers are given in Fig. 7, all 


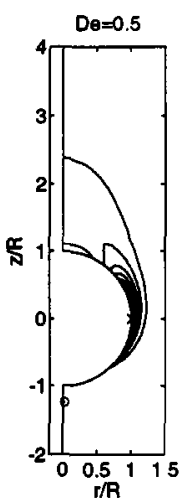

o Min: -1.07 $\times$ Max: 42.84

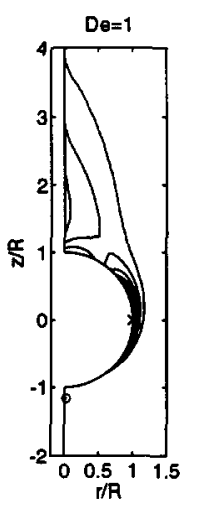

O Min: -0.7075

$\times \operatorname{Max}: 69.17$

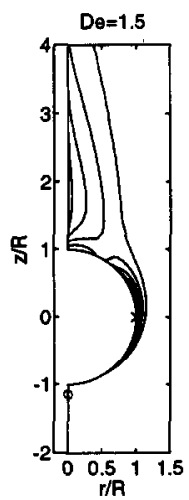

$\circ$ Min: -0.533 $\times \operatorname{Max}: 92.35$

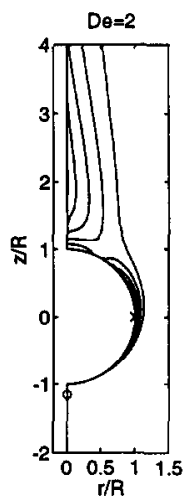

OMin: -0.4271

$\times$ Max: 113

Fig. 7. Contour lines of $\tau_{z z}$ in the vicinity of the sphere at four Deborah numbers for the constant stress element.

for mesh 4. Ten equally spaced contour lines between the minimum and maximum values are drawn. Contour lines are based on stresses obtained by a least-squares-projection of the discontinuous $P_{0}^{d}$ stress field on a continuous $P_{1}$ field. A progressive downstream shift of the contour lines and a relative narrowing of the stress boundary layer is observed with increasing Deborah number.

For quantitative comparison, Fig. 8 shows the $\tau_{z z}$ component for constant stress element along the centerline at the wake of the sphere, plotted at four values of the Deborah number. Stresses increase monotonically with increasing Deborah number and compare well with those reported by Crochet and Legat [26].

\subsection{Stick-slip problem}

The stick-slip problem is a so-called 'non-smooth' problem as it contains a geometrical singularity at the transition point from stick to slip. It is known to be notoriously difficult to solve and many algorithms show a failure of the Newton iteration process combined with strong oscillations of the stress field at moderate values of the Deborah number.

The geometry is sketched in Fig. 9. As dimensions $L_{1} / H=15$ and $L_{2} / H=40$ are chosen. A detail of the mesh near the stick-slip transition that has been used in all subsequent calculations is depicted in Fig. 10. This mesh contains 900 elements, 496 nodes and the smallest element at the singularity has a size of $h_{x} / H=0.08$ and $h_{y} / H=0.01$. 


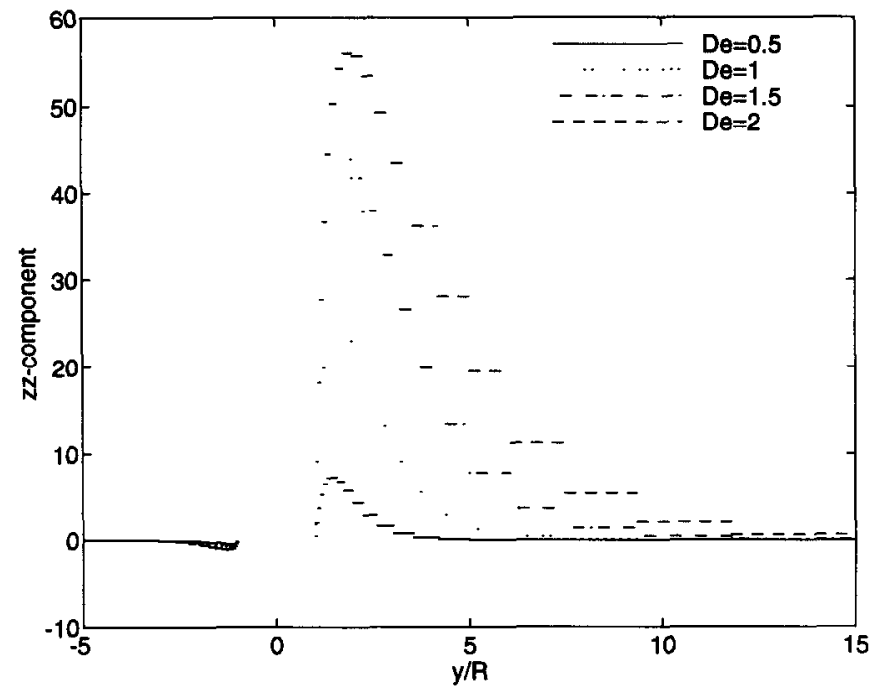

Fig. 8. The $\tau_{z z}$ stress component of the constant stress element along the symmetry line for mesh 4.

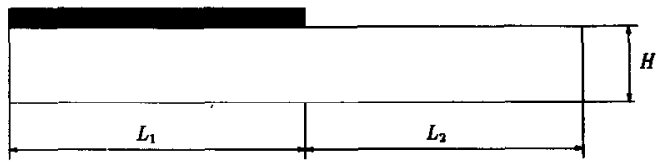

Fig. 9. Geometry of the stick-slip problem.

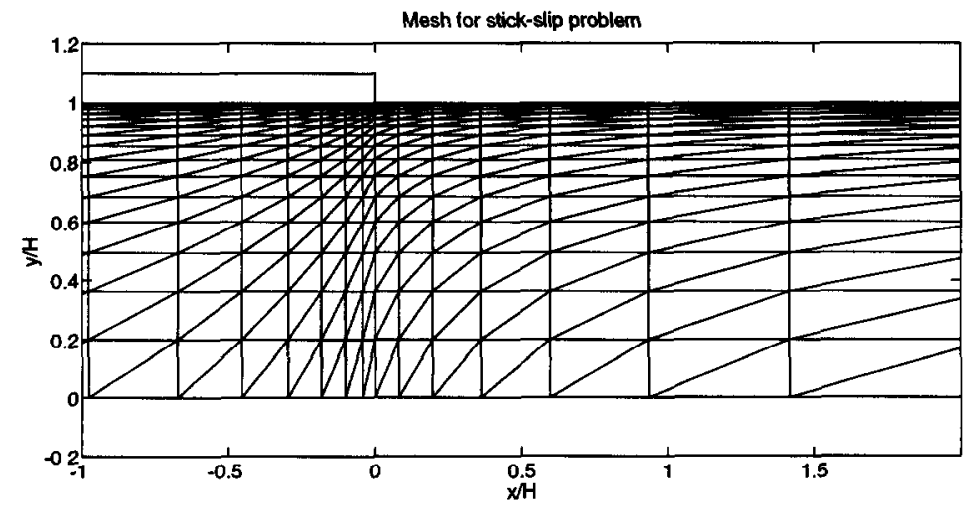

Fig. 10. Detail of the mesh near the stick-slip transition point. 
As mentioned before, the Deborah number is taken as $D e=\lambda \dot{\gamma}_{\mathrm{w}}$ with $\dot{\gamma}_{\mathrm{w}}$ the wall shear rate.

\subsubsection{Stick-slip problem with the PTT model}

First, the PTT model is employed with $\epsilon=0.25$. Computations are stopped at $D e=89$ because only little change in the stress field is observed beyond this value. In all convergent cases a quadratic rate of convergence of the Newton iteration process is noticed. The linear stress element failed to converge at much lower values of the Deborah number; see Baaijens [21].

Using the constant stress element, Fig. 11 shows the computed pressures at a range of $D e$. The viscous pressure distribution is included as a reference. In all cases a smooth pressure distribution is found, demonstrating the effectiveness of the stabilization procedure. The minima and maxima referred to in the figures are mostly located outside the domain shown in the figures.

The first normal stress coefficient $N_{1}$ along the line $y / H=1$ is drawn in Fig. 12. At the Deborah number of 89 , the effect of decreasing $\epsilon$ from 0.25 to 0.05 on this $N_{1}$ profile is shown in Fig. 13. The same behaviour was observed by Fortin et al. [15], but convergence is obtained at much higher values of the Deborah number for $\epsilon=0.05$.
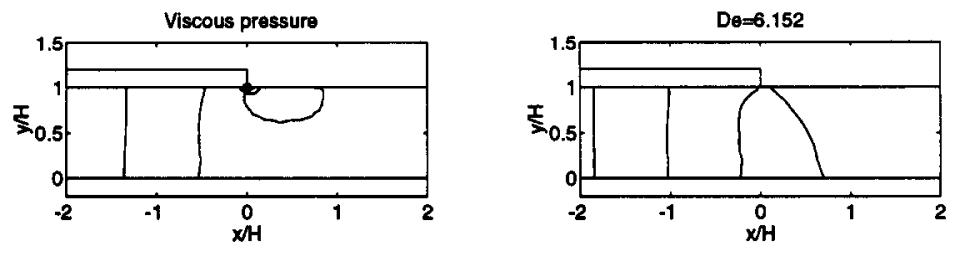

- Min: -7.949

$\times$ Max: 44.3

- Min: -0.0007699

$\times \operatorname{Max}: 21.77$
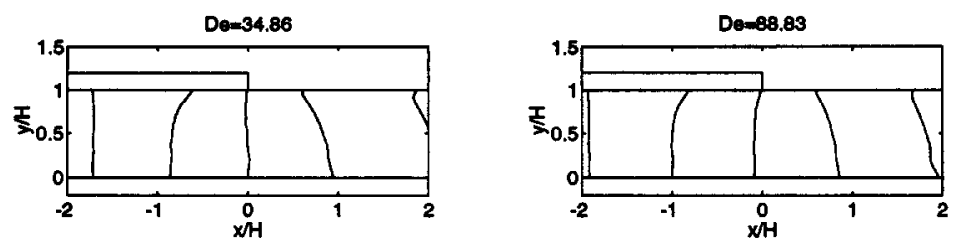

- Min: 0.002375

$\times$ Max: 9.206

- Min: $\mathbf{0 . 0 1 4 2 7}$

$\times$ Maxe 5.5

Fig. 11. Distribution of the pressure field near the singularity for the PTT model at $\epsilon=0.25$. 


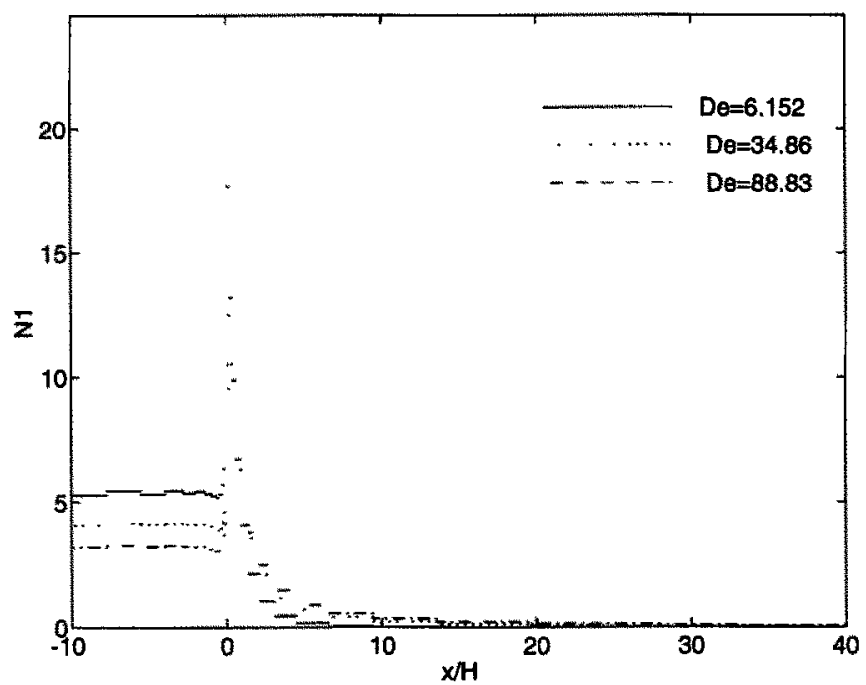

Fig. 12. The first normal stress difference $N_{1}$ along $y / H=1$ for the PTT model with $\epsilon=0.25$.

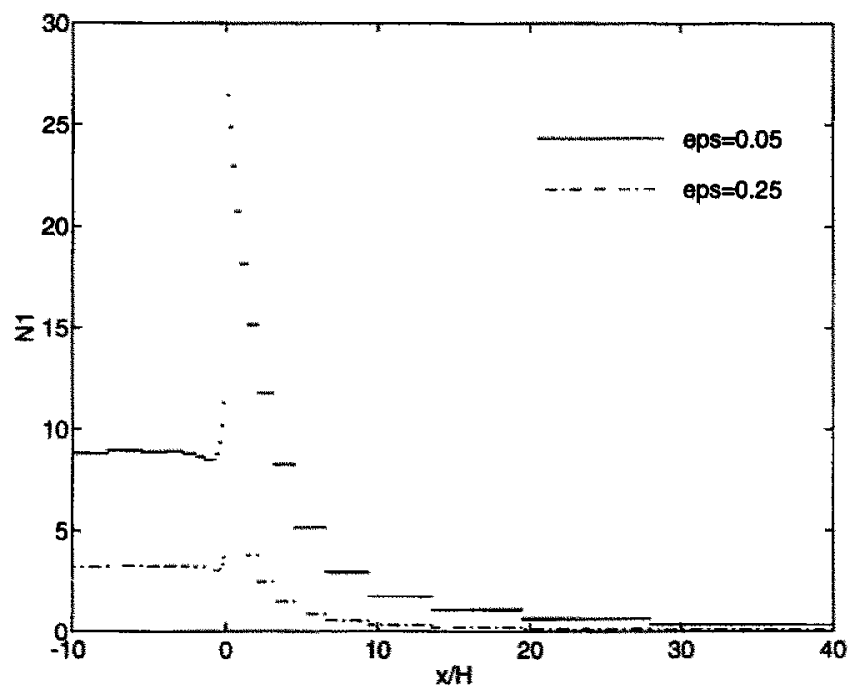

Fig. 13. The first normal stress difference $N_{3}$ along $y / H=1$ for the PTT model with $\epsilon=0.25$ and 0.05 .

\subsubsection{Stick-slip problem with the UCM model}

The stick-slip problem proves to be more difficult to solve for the UCM model. Convergence is achieved until $D e=25.5$. It is not understood, yet, what causes this loss of convergence of the iterative scheme. This may indicate a true failure of the algorithm, may be due to anomalies in the 

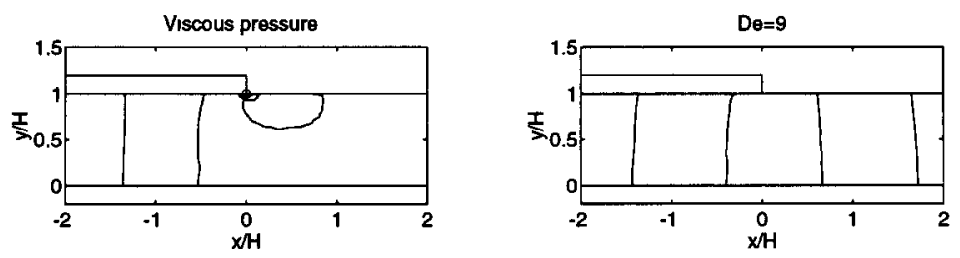

- Min: -7949

- Min: -0.02264

$\times$ Max: 44.3

$\times \operatorname{Max}: 6456$
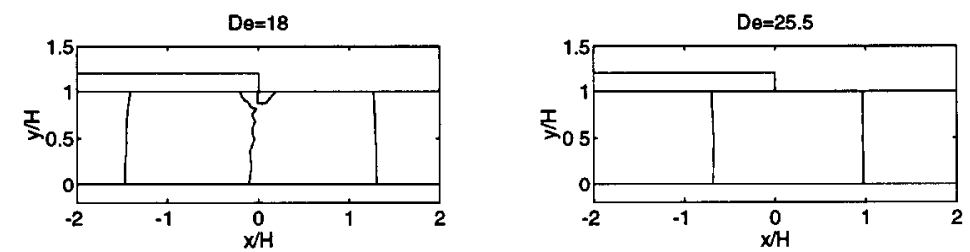

$\circ$ Min: 0.145

$\times$ Max: 85.48

- Min: 1.365

$\times \operatorname{Max} 1031$

Fig. 14. Distribution of the pressure field near the singularity for the UCM model.

UCM model (i.e. singularity in the elongational viscosity), but may also be attributed to the limited entry and exit lengths.

Figure 14 shows the computed pressure field at a sequence of Deborah numbers. As in the case of the PTT model, a smooth pressure field is observed for all values of the Deborah number, again demonstrating the

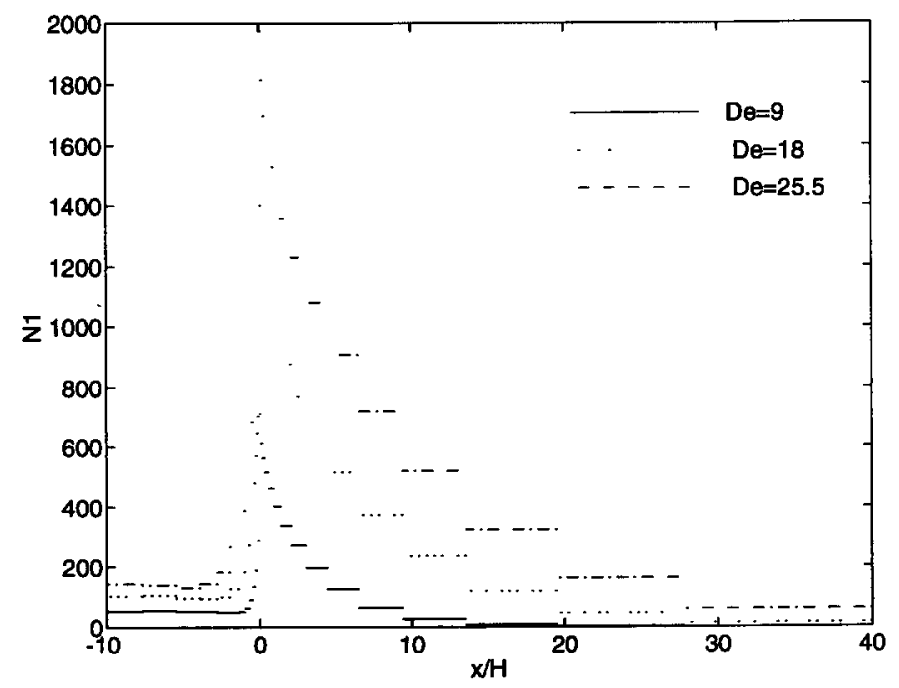

Fig. 15. The first normal stress difference $N_{1}$ along $y=1$ for the UCM model. 
effectiveness of the pressure stabilization procedure. Contour lines are shown at 20 equal intervals between minimum and maximum values.

The first normal stress difference along $y / H=1$ at a sequence of Deborah numbers is drawn in Fig. 15 and no oscillations are observed.

\section{Conclusions}

The performance of two low-order interpolation schemes (linear and constant) of the extra stress tensor in the Discontinuous Galerkin method is investigated numerically. To be able to use the constant stress interpolation in combination with a linear velocity-pressure interpolation, the Galerkinleast-squares methodology developed by Franca and Stenberg [22] is applied.

In particular with the constant stress element the Discontinuous Galerkin method exhibits a robust numerical performance, giving a convergent Newton iteration process up to high values of the Deborah number in smooth and non-smooth benchmark problems using the UCM and the PTT constitutive models.

For the falling sphere in a tube problem, using the constant stress element, convergence of the iterative scheme at least up to a Deborah number of 4 is achieved for the UCM model. For this element, convergence with mesh refinement is rather slow, in particular at elevated Deborah numbers. In fact, for the falling sphere problem using the UCM model, convergence with mesh refinement has not been established yet, as this is beyond practical limits of our software/hardware configuration. This point certainly needs to be addressed in the near future. For unsteady problems, the implicit/explicit algorithm proposed by Baaijens [21] may solve these problems partially, at the price of taking many time steps.

The linear stress element is clearly more accurate than the constant stress element, but less robust. For the falling sphere problem convergence up to $D e=1.6$ is observed but significant stress oscillations are present near and at the sphere wall.

With the constant stress element, a limiting Deborah number of 25.5 is observed for the stick-slip problem in the case of the upper convected Maxwell model, while no limit is found for the Phan-Thien-Tanner model with $\epsilon>0.05$.

The effectiveness of the pressure-stabilization procedure is clearly demonstrated by the smoothness of the pressure fields.

Finally, to achieve convergence with mesh refinement of the constant stress element, highly refined meshes are needed. However, the constant stress element need not be applied throughout the computational domain. The use of the constant stress element in 'difficult' regions in combination 
with a higher order element in 'easy' regions may lead to a computationally robust and efficient method. This topic is left for future work.

\section{References}

1 A.N. Beris, R.C. Armstrong and R.A. Brown, J. Non-Newtonian Fluid Mech., 16 (1984) 141-172.

2 J.J. van Schaftingen and M.J. Crochet, A comparison of mixed methods for solving the flow of a Maxwell fluid, Int. J. Numer. Methods Fluids, (1984) 1065-1081 4.

3 J.M. Marchal and M.J. Crochet, A new mixed finite element for calculating viscoelastic flow, J. Non-Newtonian Fluid Mech., 26 (1987) 77-114.

4 B. Debbaut, J.M. Marchal and M.J. Crochet, Numerical simulation of highly viscoelastic flows through an abrupt contraction, J. Non-Newtonian Fluid Mech., 29 (1988) 119-146.

5 R.C. King, M.R. Apelian, R.C. Armstrong and R.A. Brown, Numerically stable finite element techniques for viscoelastic calculations in smooth and singular domains, J. Non-Newtonian Fluid Mech., 29 (1988) 147-216.

6 D. Rajagopalan, R.C. Armstrong and R.A. Brown, Calculation of steady viscoelastic flow using a multimode Maxwell model: application of the explicitly elliptic momentum equation (EEME) formulation, J. Non-Newtonian Fluid Mech., 36 (1990) 135-157.

7 D. Rajagopalan, R.C. Armstrong and R.A. Brown, Finite element methods for calculation of steady viscoelastic flow using constitutive equations with a Newtonian viscosity, J. Non-Newtonian Fluid Mech., 26 (1990) 159-192.

8 P.J. Coates, R.C. Armstrong and R.A. Brown, Calculation of steady-state viscoelastic flow through axisymmetric contractions with the EEME formulation, J. Non-Newtonian Fluid Mech. 42 (1992) 141-188.

9 X.L. Luo and R.I. Tanner, A decoupled finite element streamline-upwind scheme for viscoelastic flow problems, J. Non-Newtonian Fluid Mech., 31(1989) 143-162.

10 F.P.T. Baaijens, Numerical analysis of unsteady viscoelastic flow, Comput. Methods Appl. Mech. Eng., 94 (1992) 285-299.

11 J. Baranger and D. Sandri, A formulation of Stokes' problem and the linear elasticity equations suggested by the Oldroyd model for viscoelastic flow, Math. Modelling Numer. Anal. 26 (1992) 331-345.

12 L.A. Ying, Comput. Mech., 2 (1987) 45-53.

$13 \mathrm{M}$. Fortin and R. Pierre, On the convergence of the mixed method of Crochet and Marchal for viscoelastic flows, Int. J. Numer. Methods Fluids, 7 (1987) 1035-1052.

14 M. Fortin and A. Fortin, A new approach for the FEM simulation of viscoelastic flows, J. Non-Newtonian Fluid Mech., 32 (1989) 295-310.

15 A. Fortin, A. Zine and J.-F. Agassant, Computing viscoelastic fluid flow problems at low cost, J. Non-Newtonian Fluid Mech., 42 (1992) 1-8.

16 F.P.T. Baaijens, Numerical analysis of start up planar and axisymmetric contraction flows using multi-mode differential constitutive models, J. Non-Newtonian Fluid Mech., 48 (1993) 147-180.

17 F.P.T. Baaijens, An U-ALE formulation of 3-D unsteady viscoelastic flow, Int. J. Numer. Methods Eng., 36 (1992) 1115-1143.

18 M.A. Mendelson, P.W. Yeh, R.C. Armstrong and R.A. Brown, J. Non-Newtonian Fluid Mech., 10 (1982) 31-54.

19 F.G. Basombrio, G.C. Buscaglia and E.A. Dari, Simulation of highly elastic fluids without additional numerical diffusivity, J. Non-Newtonian Fluid Mech., 39 (1991) 189-206. 
20 O. Pironneau, Finite Element Method for Fluids, John Wiley, New York, 1989.

21 F.P.T. Baaijens, Numerical experiments with a Discontinuous Galerkin method including monotonicity enforcement on the stick-slip problem, 1993, J. Non-Newtonian Fluid Mech., 51 (1994) 141-159.

22 L. Franca and R. Stenberg, Error analysis of some Galerkin-Least-Squares methods for the elasticity equations, SIAM J. Numer. Anal., 28 (1991) 1680-1697.

23 M.A. Behr, L.P. Franca and T.E. Tezduryar, Stabilized finite element methods for the velocity-pressure-stress formulation of incompressible flows, Comput. Methods Appl. Mech. Eng., 104 (1993) 31-48.

24 T.E. Tezduyar, S. Mittal, S.E. Ray and R. Shih, Incompressible flow computations with stabilized bilinear and linear equal-order-interpoaltion velocity-pressure elements, Comput. Methods Appl. Mech. Eng., 95 (1992) 221-242.

25 T.J.R. Hughcs, L.P. Franca and M. Balestra, A new finite element formulation for computational fluid dynamics. V. Circumventing the Babuska-Brezzi condition: a stable Petrov-Galerkin formulation of the Stokes problem accommodating equal-order interpolations, Computer. Methods Appl. Mech. Eng., 59 (1986) 85-99.

26 M.J. Crochet and V. Legat, The consistent streamline-upwind/Petrov-Galerkin method for viscoelastic flow revisited, J. Non-Newtonian Fluid Mech., 42 (1992) 283-299.

27 H.K. Rasmussen and O. Hassager, Simulation of transient viscoelastic flow, J. Non-Newtonian Fluid Mech., 46 (1993) 298-305.

28 M.D. Chilcott and J.M. Rallison, Creeping flow of dilute polymer solutions past cylinders and spheres, J. Non-newtonian Fluid Mech., 29 (1988) 381-432.

29 O.G. Harlen, High-Deborah number flow of a dilute polymer solution past a sphere falling along the axis of a cylindrical tube, J. Non-Newtonian Fluid Mech., 37 (1990) 157-173.

30 W.J. Lunsmann, L. Genieser, R.C. Armstrong and R.A. Brown, Finite element analysis of steady viscoelastic flow around a sphere in a tube: calculations with constant viscosity models, J. Non-Newtonian Fluid Mech., (1993) 63-99.

31 R.C. Armstrong, M.R. Apelian and R.A. Brown, Impact of the constitutive equation and singularity on the calculation of stick-slip flow: the modified upper-convected Maxwell model (MUCM), J. Non-Newtonian Fluid Mech., 27 (1988) 299-321.

32 A. Fortin and M. Fortin, A preconditioned generalized minimal residual algorithm for the numerical solution of viscoelastic flows, J. Non-Newtonian Fluid Mech., 36 (1990) $227-288$.

33 J. van der Zanden and M.A. Hulsen, Mathematical and physical requirements for successful computations with viscoelastic fluid models, J. Non-Newtonian Fluid Mech., (1988) 93.

34 L. Franca and T.J.R. Hughes, Two classcs of mixed finite element methods, Compute Methods Appl. Mech. Eng., 69 (1988) 89-129.

35 Y. Saad and M.H. Schultz, GMRES: a generalized minimal residual algorithm for solving nonsymmetric linear system, SIAM J. Sci. Stat. Comput., 7 (1986) 856869.

36 J.R. Gilbert, C. Moler and R. Schreiber, Sparse matrices in MATLAB: design and implementation, SIAM J. Matrix Anal. Appl., 13 (1992) 333-356.

37 R.I. Tanner and H. Jin, A study of some numerical viscoelastic schemes, J. Non-Newtonian Fluid Mech., (1992) 171-196.

38 J. Happel and H. Brenner, Low Reynolds Number Hydrodynamics, Mantinus Nijhoff, New York, 1986. 